Letter

\title{
Serum C3 Enhances Complement Receptor 3-Mediated Phagocytosis of Borrelia burgdorferi
}

\author{
Kelly L. Hawley ${ }^{1 *}$, Chris M. Olson, Jr. ${ }^{1 \#, ~ A n a ~ C a r r e r a s-G o n z a ́ l e z ~}{ }^{2}$, Nicolás Navasa 2, Juan Anguita 1,2,3凶 \\ 1. Department of Veterinary and Animal Sciences, University of Massachusetts Amherst, 01003 Amherst, MA, USA \\ 2. CIC bioGUNE, Bizkaia Technology Park, 48160 Derio, Spain \\ 3. IKERBASQUE, Basque foundation for science, 48013 Bilbao, Spain \\ * KLH present address: UCONN Health Center, 263 Farmington Ave. Farmington, CT \\ \# CMOJ present address: Mayo Medical School, 200 First St SW. Rochester, MN. \\ $\triangle$ Corresponding author: janguita@cicbiogune.es
}

() 2015 Ivyspring International Publisher. Reproduction is permitted for personal, noncommercial use, provided that the article is in whole, unmodified, and properly cited. See http://ivyspring.com/terms for terms and conditions.

Received: 2015.07.30; Accepted: 2015.09.03; Published: 2015.09.11

Complement receptor $(\mathrm{CR}) 3$ is a bona fide phagocytic receptor for Borrelia burgdorferi, the causative agent of Lyme borreliosis [1, 2]. CR3 is also the receptor for the opsonin $\mathrm{iC} 3 \mathrm{~b}$, a final degradation product of the complement component C3 $[3,4]$. The transit of $B$. burgdorferi through the blood during the dissemination phase exposes the spirochete to serum components, including complement [5]. B. burgdorferi contains proteins with anti-complement activities that trigger the inactivation of $\mathrm{C} 3 \mathrm{~b}$ through the binding of factor $\mathrm{H}(\mathrm{FH})$ on the surface of the bacterium [6]. This could lead to the accumulation of the opsonin, $\mathrm{iC} 3 \mathrm{~b}$, and the enhanced phagocytosis of the spirochete. To test whether the presence of serum would increase the phagocytosis of $B$. burgdorferi, we incubated bone marrow-derived macrophages (BMMs), generated as described [1], with heat-inactivated (HI; $\left.56^{\circ} \mathrm{C}, 30 \mathrm{~min}\right)$ or active normal mouse sera (NMS). Phagocytosis protocols were followed according to published reports $[1,2]$. The presence of $10 \% \mathrm{HI}$ serum resulted in phagocytosis levels comparable to those in the absence of serum (Fig. 1A,B). However, the presence of $10 \%$ NMS enhanced the capacity of macrophages to internalize B. burgdorferi (Fig. 1A). The same effect was observed on RAW264.7 (RAW) cells (Fig. 1C). Thus, the contact of $B$. burgdorferi with serum components results in their enhanced phagocytic uptake. Next, we assessed whether the increased phagocytic activity associated with the presence of serum was due to C3-derived opsonin generation. We used sera from C3-deficient mice ( $\mathrm{C} 3 \mathrm{KO})$, in which $B$. burgdorferi dissemination is enhanced compared to WT mice [5]. The presence of C3 KO serum abrogated the increased phagocytosis observed in the presence of NMS, both in BMMs (Fig. 1A,B) and RAW cells (Fig. 1C).

To demonstrate that the enhanced phagocytosis of $B$. burgdorferi in the presence of NMS was specific to CR3, we used $\mathrm{CHO}$ cells transfected with human CR3 (CD11b/CD18; CHO-CR3) or CR4 (CD11c/CD18; $\mathrm{CHO}-\mathrm{CR} 4)$ as controls $[1,7]$. Incubation of $\mathrm{CHO}-\mathrm{CR} 3$, but not $\mathrm{CHO}-\mathrm{CR} 4$, cells resulted in increased binding of the spirochete (Fig. 2A). Furthermore, the presence of a blocking CD11b antibody (M1/70) [1] also resulted in the reduction of B. burgdorferi phagocytosis in the presence of NMS in RAW cells (Fig. 2B). We also used BMMs generated from CD11b KO mice. Consistently, the presence of NMS during phagocytosis failed to increase the phagocytosis of B. burgdorferi in the absence of CD11b, compared to the use of HI serum (Fig. 2C), demonstrating that sera increased the phagocytosis of B. burgdorferi through its interaction with CR3.

We have demonstrated that CR3-mediated phagocytosis tempers the inflammatory output of macrophages [1]. We argued that the CR3-dependent boost in phagocytosis in the presence of NMS would also reduce the production of TNF in response to $B$. burgdorferi. We stimulated BMMs with live $B$. burgdorferi at an m.o.i. of 25 for $16 \mathrm{~h}$ in the presence of NMS or C3-deficient serum. The presence of NMS significantly reduced the production of TNF by BMMs (Fig. 2D). Importantly, the absence of $\mathrm{C} 3 \mathrm{ab}-$ 
rogated this inhibitory effect (Fig. 2D). The effect of active serum on macrophages was specific for TNF, since the levels of IL- 6 did not change in the presence of active serum (Fig. 2E). Furthermore, the effect was specific of ligands that interact with CR3, since the presence of serum did not result in changes in the levels of TNF produced by BMMs in response to the TLR4 agonist, LPS (Fig. 2F). Overall, these data demonstrate that CR3 is an anti-inflammatory phagocytic receptor both in the absence [1] and the presence of the opsonin iC3b.

In summary, our results show that serum C3-derived opsonins enhance the phagocytosis of $B$. burgdorferi mediated by CR3 further tempering the inflammatory output of macrophages induced by the integrin.
A

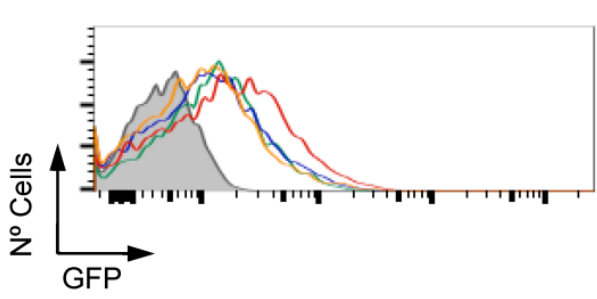

B

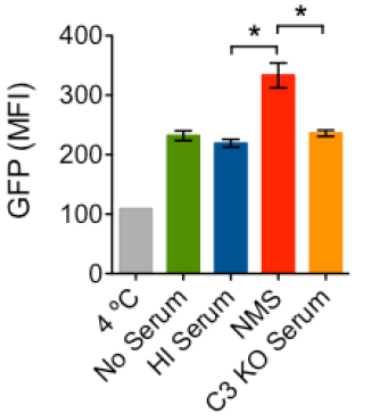

C

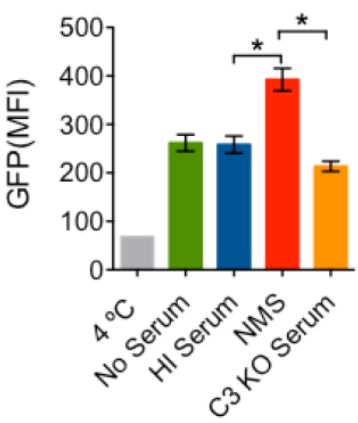

Figure 1. Serum increases phagocytosis of B. Burgdorferi in a C3-dependent manner. (A) BMMs were incubated with GFP-expressing B. burgdorferi (Bb914) at an m.o.i. of 50, in the presence of $10 \% \mathrm{HI}$ (blue histogram), NMS (red histogram), C3-deficient serum (orange histogram) or without serum (green histogram). Phagocytosis was allowed to occur for $4 \mathrm{~h}$ (BMMs) or $6 \mathrm{~h}$ (RAW cells), followed by analysis by flow cytometry. The data were analyzed using Flowjo version 10. The average mean fluorescence intensity was evaluated in triplicates for BMMs (B) and RAW cells (C). The grey histogram/bars represent a $4{ }^{\circ} \mathrm{C}$ control. The data presented represent one representative experiment of at least 3 performed.

A

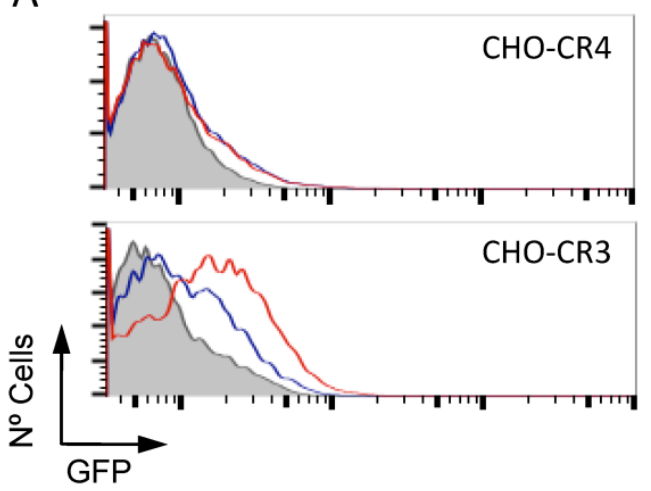

B

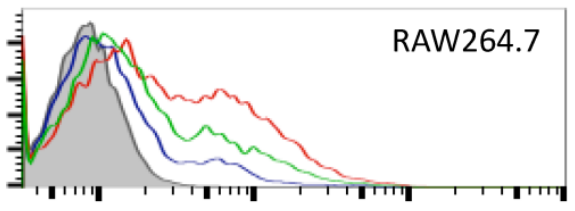

C

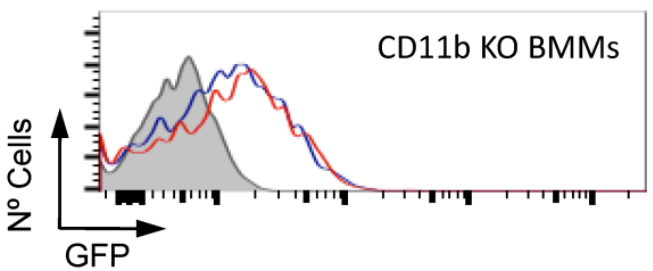

D

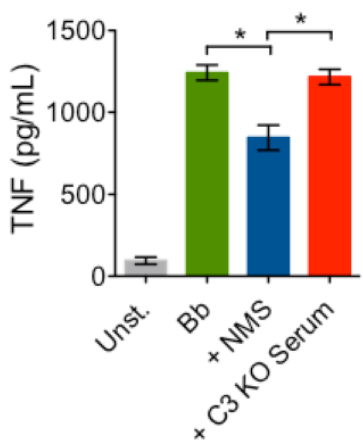

$\mathrm{E}$

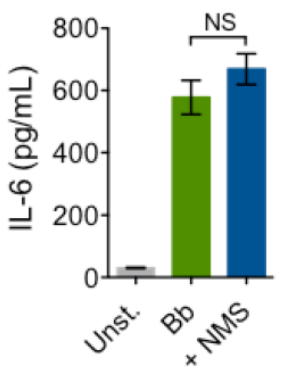

$\mathrm{F}$

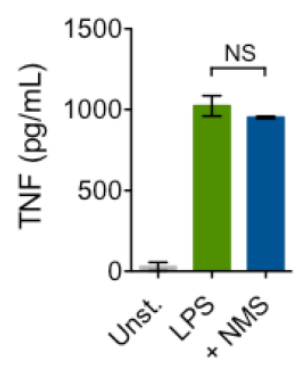

Figure 2. Serum-mediated increase of B. Burgdorferi phagocytosis is CR3 dependent. (A) CHO-CR3 (bottom panel) and CHO-CR4 (top panel) cells were incubated with Bb914 (m.o.i. = 50) in the presence of $\mathrm{HI}$ (blue histogram) or NMS (red histogram). After $6 \mathrm{~h}$, the binding of B. burgdorferi was determined by flow cytometry. (B) RAW cells were incubated with Bb914 in the presence of $\mathrm{HI}$ (blue histogram), NMS (red histogram) or NMS $+10 \mu g / \mathrm{mL}$ of a blocking CDI lb antibody (green histogram). After $2 \mathrm{~h}$, the cells were analyzed by flow cytometry. (C) CDI l b-deficient BMMs were incubated with Bb914 in the presence of HI (blue histogram) or NMS (red histogram). The cells were analyzed by flow cytometry after $4 \mathrm{~h}$. The grey histograms represent a $4{ }^{\circ} \mathrm{C}$ control. The data represent one of at least 3 independent experiments performed in triplicate. (D) BMMs from $\mathrm{C} 57 \mathrm{BI} / 6$ mice were stimulated with live B. Burgdorferi $(\mathrm{m} .0 . \mathrm{i} .=25)$ in the presence of NMS (blue bar) or C3-deficient serum (red bar). TNF levels in the stimulation supernatants were measured $16 \mathrm{~h}$ later by ELISA. (E, F) BMMs were stimulated with B. burgdorferi (m.o.i. = 25) or LPS $(100 \mathrm{ng} / \mathrm{mL})$ in the presence of HI (green bar) or NMS (blue bar) for $16 \mathrm{~h}$. The supernatants were assessed for IL-6 (E) and TNF (F) by ELISA. 


\section{Acknowledgements}

Supported by grants from the Spanish Ministry of Economy and Competitiveness (MINECO, SAF2012-34610), the American Heart Association (CMOJ) and a fellowship from the Basque Government (AC).

We thank Justin D. Radolf for providing the GFP-expressing B. burgdorferi and Erol Fikrig and Sam Black for generously providing C3-deficient sera.

\section{Competing Interests}

The authors have declared that no competing interest exists.

\section{References}

1. Hawley KL, Olson CM, Jr., Iglesias-Pedraz JM, Navasa N, Cervantes JL, Caimano MJ, Izadi H, Ingalls RR, Pal U, Salazar JC et al: CD14 cooperates with complement receptor 3 to mediate MyD88-independent phagocytosis of Borrelia burgdorferi. Proc Natl Acad Sci U S A 2012, 109:1228-32.

2. Hawley KL, Martin-Ruiz I, Iglesias-Pedraz JM, Berwin B, Anguita J: CD14 targets complement receptor 3 to lipid rafts during phagocytosis of Borrelia burgdorferi. Int J Biol Sci 2013, 9:803-10.

3. Le Cabec V, Carreno S, Moisand A, Bordier C, Maridonneau-Parini I: Complement receptor 3 (CD11b/CD18) mediates type I and type II phagocytosis during nonopsonic and opsonic phagocytosis, respectively. J Immunol 2002, 169:2003-9.

4. Ueda T, Rieu P, Brayer J, Arnaout MA: Identification of the complement iC3b binding site in the beta 2 integrin CR3 (CD11b/CD18). Proc Natl Acad Sci U S A 1994, 91:10680-4.

5. Lawrenz MB, Wooten RM, Zachary JF, Drouin SM, Weis JJ, Wetsel RA, Norris SJ: Effect of complement component C3 deficiency on experimental Lyme borreliosis in mice. Infect Immun 2003, 71:4432-40.

6. Hellwage J, Meri T, Heikkila T, Alitalo A, Panelius J, Lahdenne P, Seppala IJ, Meri S: The complement regulator factor $\mathrm{H}$ binds to the surface protein OspE of Borrelia burgdorferi. J Biol Chem 2001, 276:8427-35.

7. Medvedev AE, Flo T, Ingalls RR, Golenbock DT, Teti G, Vogel SN, Espevik T: Involvement of CD14 and complement receptors CR3 and CR4 in nuclear factor-kappaB activation and TNF production induced by lipopolysaccharide and group B streptococcal cell walls. J Immunol 1998, 160:4535-42. 